\title{
Terahertz Digital Holographic Imaging of Voids Within Visibly Opaque Dielectrics
}

\author{
Martin S. Heimbeck, Wei-Ren Ng, Dathon R. Golish, Michael E. Gehm, and Henry O. Everitt
}

\begin{abstract}
Terahertz digital off-axis holography (THzDH) has been demonstrated as a non-destructive tool for imaging voids within visually opaque dielectrics. Using a raster scanning heterodyne detector, the imager captures lensless transmission holograms formed by the interaction of a highly coherent, monochromatic beam with 3-D printed structures. Digital hologram reconstructions from two structures were used to measure the imager's modulation transfer function and to show that terahertz digital holography can provide sub-millimeter resolution images of voids within visually opaque printed structures. As a demonstration we imaged embedded air- and lossy dielectric filled-voids whose refractive indices differ from the host material.
\end{abstract}

Index Terms-Holography, submillimeter-wave measurements, submillimeter-wave propagation.

\section{INTRODUCTION}

$\mathbf{T}$ ERAHERTZ digital holography (THzDH), analogous to its visible wavelength counterpart, combines phase-sensitive optical interferometric imaging methods with computational holographic reconstruction and processing algorithms. By applying lensless digital holography at $\mathrm{THz}$ frequencies, the three dimensional interior of visibly opaque dielectrics may be imaged with sub-millimeter transverse and axial resolution and without the need of physically defining a focal plane inside the object [1]-[6]. The digitally recorded hologram can be reconstructed, quantitatively analyzed, and enhanced using computational techniques, such as digital filtering, digital field propagation, and multi-wavelength hologram overlays to extend the phase unambiguous range [3], [7], [8].

Although $\mathrm{THz}$ imaging based on spatially and temporally scanned pulsed laser techniques is quite mature, the time required to record an image can vary from minutes to days depending on the application and desired resolution. The lateral resolution of such systems is limited by the size of the focused spot, while the axial resolution is determined by the bandwidth

Manuscript received June 10, 2014; revised August 29, 2014; accepted October 14, 2014. Date of publication November 11, 2014; date of current version January 14, 2015.

M. S. Heimbeck is with the Charles M. Bowden Research Center, Army Aviation \& Missile RD\&E Center, Redstone Arsenal, AL 35898 USA.

W. Ng and D. R. Golish are with the Department of Electrical and Computer Engineering, University of Arizona, Tucson, AZ 85721 USA.

M. E. Gehm is with the Department of Electrical and Computer Engineering, Duke University, Durham, NC 27708 USA.

H. O. Everitt is with the Charles M. Bowden Center, Army Aviation \& Missile RD\&E Center, Redstone Arsenal, AL 35898 USA, and also with the Department of Physics, Duke University, Durham, NC 27708 USA (e-mail: everitt@phy. duke.edu)

Color versions of one or more of the figures in this paper are available online at http://ieeexplore.iee.org

Digital Object Identifier 10.1109/TTHZ.2014.2364511 of the pulse and the temporal scanning fidelity. By contrast, holographic imaging can produce high quality, high resolution images quickly using detector arrays without forming a focal spot that must be scanned across the object. Instead, lateral resolution is determined interferometrically by the spatial fringes, detector aperture, and beam splitter angle, while the axial resolution is determined by fringe visibility and detector dynamic range [1]-[7]. Although arrays of sensitive $\mathrm{THz}$ detectors are not yet available, it is not too soon to explore the foundational tenets of THzDH using scanned, single pixel detectors.

For these reasons, THzDH is emerging as a promising technique for applications including security screening and non-destructive test and evaluation (NDT\&E) [9]-[12]. Of particular interest for NDT\&E is the detection and spatial mapping of inclusions embedded within visually opaque hosts. Here we report the application of $\mathrm{THzDH}$ using a single, raster-scanned detector to acquire a digital hologram and reconstruction algorithms to detect and map the transverse locations of polymer structures and air voids in visually opaque dielectric samples. 3D printing technology - which has already been used to fabricate intricate dielectric $\mathrm{THz}$ lenses, waveguides, and spatial light modulators [9], [13]-[16] — was used to fabricate two voidcontaining samples. The first (sample A) was used to illustrate the capabilities of the holographic imager by measuring its modulation transfer function and showing how well the technique can differentiate between printed materials with slightly different complex refractive indices. The imager's ability to locate 400 air voids within an object is illustrated with sample B, in which the strength of the associated Fabry-Pérot resonances varies with void thickness (from 15 to $6000 \mu \mathrm{m}$ ) and frequency. Generalization of these findings to new forms of NDT\&E is explored.

\section{EXPERIMENT}

A Stratasys Eden 350 3D printer fabricated the visually opaque samples containing various types of voids (Fig. 1). The samples were made from Objet VeroBlackPlus RGD875 [black areas in Fig. 1(a), (b)], while the void regions (white areas) were either empty or contained the support material Objet Support SUP705. Table I summarizes the optical properties of these two dielectrics, measured at 0.495 and $0.710 \mathrm{THz}$ with a $\mathrm{THz}$ time-domain spectroscopy system. The real refractive indices differ slightly, while the absorption coefficient of the SU705 support material is more than three times higher than that of the RGD875 model material.

Sample A [Fig. 1(a)] included nine 1-mm-thick SUP705filled void structures, capped above and below by $1 \mathrm{~mm}$ of RGD875 for a total object thickness of $3 \mathrm{~mm}$. Similar to a 

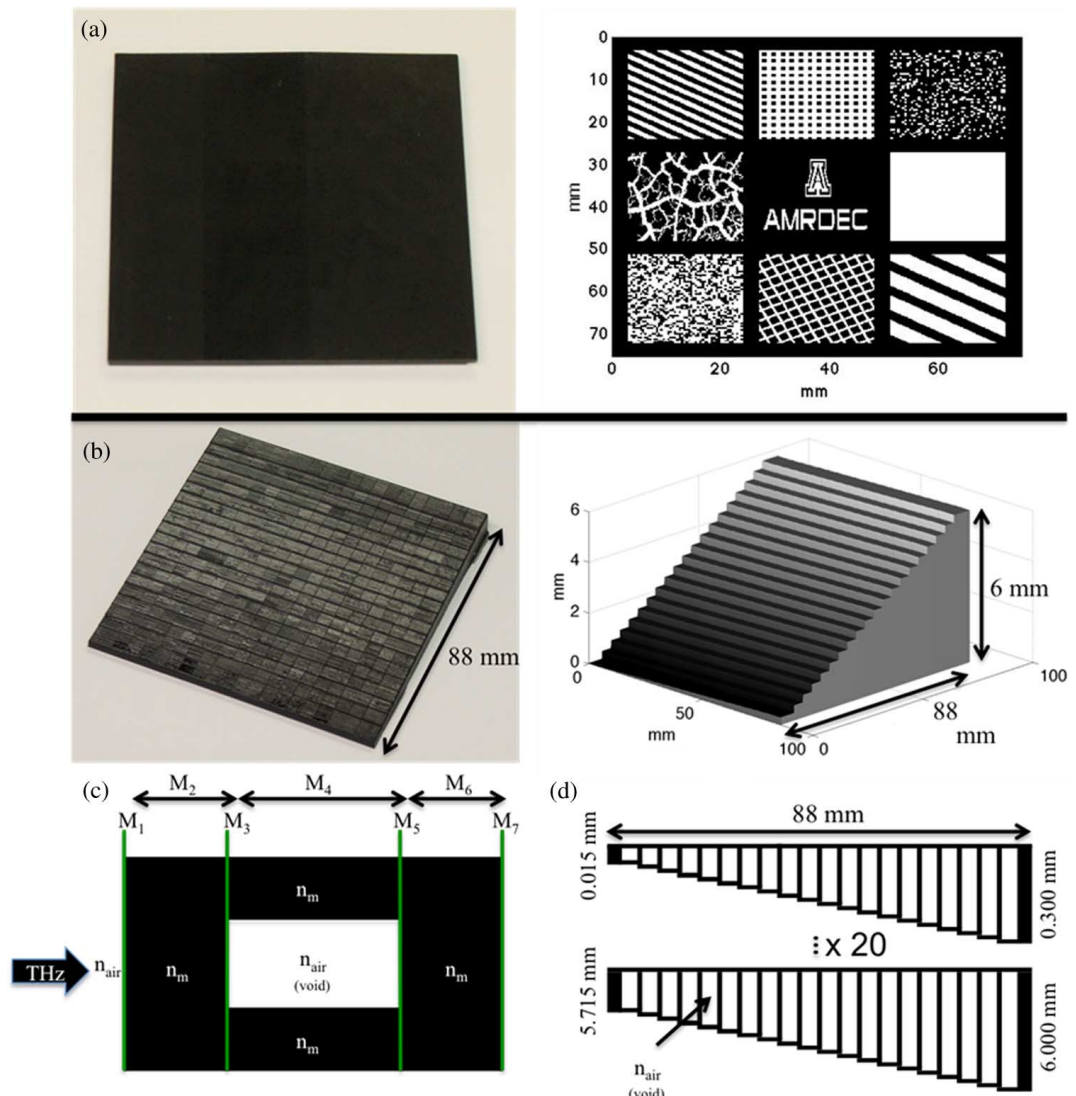

Fig. 1. (a), (b) Photographs and printing mask of 3D printed samples A and B. (c) Dielectric layers and boundaries of embedded air void sample, and (d) $x-z$ cross-sectional cut with z-axis exaggerated to illustrate the increasing air void depth ranging from 15 to $6000 \mu \mathrm{m}$ over 400 voids.

TABLE I

Optical PRoperties of Printed MATERials

\begin{tabular}{llcc}
\hline \multicolumn{1}{c}{ Material } & \multicolumn{1}{c}{ Properties } & $0.495 \mathrm{THz}$ & $0.710 \mathrm{THz}$ \\
& Refractive index $\mathrm{n}_{\mathrm{m}}$ & 1.70 & 1.70 \\
$\begin{array}{l}\text { Objet VeroBlackPlus } \\
\text { RGD875 }\end{array}$ & $\begin{array}{l}\text { Absorption } \\
\text { coefficient } \alpha_{\mathrm{m}}\left(\mathrm{cm}^{-1}\right)\end{array}$ & 0.60 & 0.75 \\
& Refractive index $\mathrm{n}_{\mathrm{S}}$ & 1.76 & 1.75 \\
$\begin{array}{l}\text { Objet Support } \\
\text { SUP705 }\end{array}$ & $\begin{array}{l}\text { Absorption } \\
\text { coefficient } \alpha_{\mathrm{s}}\left(\mathrm{cm}^{-1}\right)\end{array}$ & 2.00 & 2.50 \\
&
\end{tabular}

resolution chart, sample A allows us to measure spatial image resolution at two terahertz frequencies and to explore $\mathrm{THzDH}$ as a nondestructive imaging tool for composite dielectrics. By contrast, sample B [Fig. 1(b)] contained $4003 \mathrm{~mm} \times 3 \mathrm{~mm}$ air-filled square voids that were sequentially varied in depth from $15 \mu \mathrm{m}$ to $6000 \mu \mathrm{m}$ in $15 \mu \mathrm{m}$ steps to induce Fabry-Pérot cavity effects. The sample's top and bottom caps were $2 \mathrm{~mm}$ thick, the wall thickness between voids was $1 \mathrm{~mm}$ wide, and the frame was $2 \mathrm{~mm}$ wide. Sample B allows us to investigate the imager's ability to locate air voids of different thicknesses at the two frequencies of 0.495 and $0.710 \mathrm{THz}$.
To improve amplitude and phase resolution, the digital off-axis $\mathrm{THz}$ holographic imager [Fig. 2(a)] has been modified extensively over a version described previously in [4]. The THz source uses a Virginia Diodes, Inc. (VDI), Charlottesville, VA, USA, multiplier chain driven by a $10-20 \mathrm{GHz}$ microwave synthesizer which has the necessary amplitude and phase stability required to acquire a hologram using a raster scanned single detector. For improved dynamic range, the hologram was recorded with a heterodyne receiver instead of a Schottky diode square law detector. The receiver uses a similar VDI multiplier chain in the local oscillator to down-convert the $\mathrm{THz}$ signal in a mixer to a $1.35 \mathrm{GHz}$ intermediate frequency signal that was pre-amplified and recorded with National Instruments PXI data acquisition instrumentation. While the Schottky diode detector used in [4] routinely achieves a noise equivalent power $\mathrm{NEP} \approx-82 \mathrm{dBm} \mathrm{Hz}^{-1 / 2}$, the heterodyne receiver achieves a $\mathrm{NEP} \approx-164 \mathrm{dBm} \mathrm{Hz}^{-1}$. For the experiment, the receiver's noise floor was $-120 \mathrm{dBm}$ at a video bandwidth of $20 \mathrm{kHz}$, which was sufficient for an experiment representative signal power of $-80 \mathrm{dBm}$ at the receiver, producing a dynamic range of $40 \mathrm{~dB}$. The effective time constant for each pixel is $\sim 30 \mathrm{~ms}$.

$\mathrm{THz}$ transmission digital holograms, at 0.495 and $0.710 \mathrm{THz}$, were recorded by $2-\mathrm{D}$ raster scans of the receiver across the image plane. The optical train between the source and receiver includes a $90^{\circ}$ off-axis parabolic mirror, to collimate the diverging source beam to a diameter of approximately $20 \mathrm{~cm}$, and a $0.038-\mathrm{mm}$-thick Mylar beam splitter coated with a thin layer of metallic spray paint that equally divides the source beam 

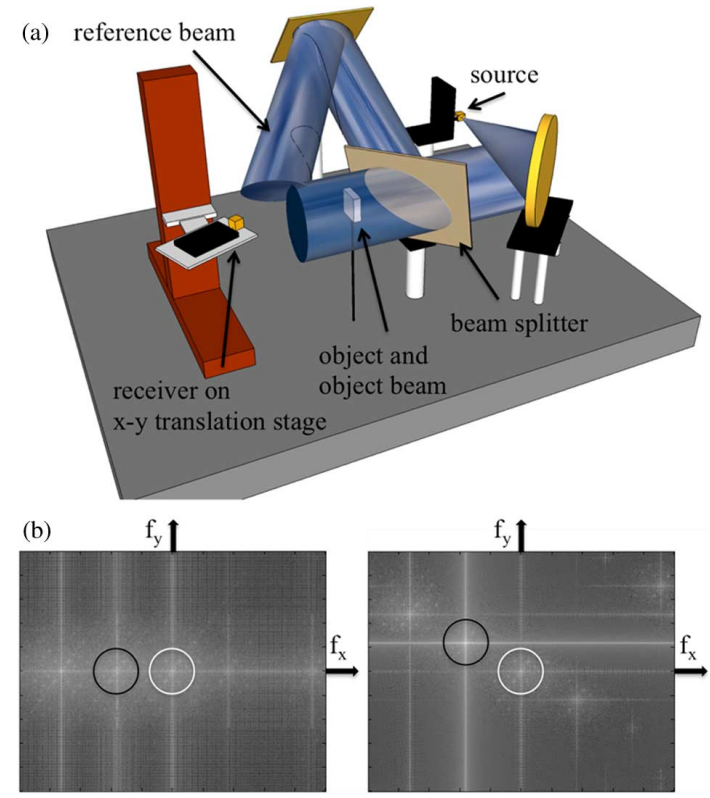

Fig. 2. Schematic diagram of the holographic imager (not to scale). (a) The blue cylinders represent the object and reference $\mathrm{THz}$ beams which produce an interferogram at the receiver. Simulated spatial frequency content of a hologram recorded with planar (left) and non-planar (right) off-axis holographic imagers. (b) The spatial frequencies indicated by the black and white circles represent the autocorrelation term and the real image, respectively, from a simulated sample.

into object and reference beams. In transmission holography, the object beam is modulated by the sample and interferes with the reference beam at the image plane where the hologram is recorded. The samples were mounted on a standard lens post and placed in the center of the object beam. Holograms were recorded by raster scanning the receiver at a distance of 163 $\mathrm{mm}$ from the sample to minimize the sample-to-detector distance, thereby maximizing image resolution without clipping the reference beam. The modulated object beam is related to the sample transfer function by scalar diffraction theory, so the holograms can be reconstructed and analyzed using the angular spectrum method (ASM) [3], [17]. Two Fourier transforms are required for the ASM, so it is more computationally intensive than the widely used Fresnel transform method, but it has several advantages including frequency spectrum filtering without constraints on the axial location of the hologram and object planes [3]. Because the reconstruction algorithm assumes that planar wavefronts illuminate the sample, baseline holograms were recorded without a sample in the object beam so that the spatial variations of amplitude and phase in the imperfect illuminating beams could be measured and corrected in a manner analogous to astronomical flat-field correction. Details of this methodology are provided in [4].

In order to achieve the smallest possible pixel size and highest spatial resolution, the receiver input was simply the open ended rectangular waveguide of the mixer with dimensions $300 \mu \mathrm{m} \times 600 \mu \mathrm{m}$. One hologram image is composed of $1000 \times 1000$ pixels with a $0.2-\mathrm{mm}$ step size for a total hologram dimension of $20 \times 20 \mathrm{~cm}$ that required $>24$ hours to acquire. Adding the usual horn antenna would have increased the dynamic range of the imager and shortened the imaging time; however, the increased pixel size and reduced maximum measurable spatial interference frequency between the reference and object beams would have made it more difficult to separate the real and virtual images from the autocorrelation term during hologram reconstruction.

To increase this separation further, a non-planar interferometer was constructed with azimuth and elevation angles between the object and reference beams of $\theta=49.2^{\circ}$ and $\varphi=23.1^{\circ}$, respectively. Although this experimental arrangement is more complicated than a planar configuration $(\varphi=0)$, the nonplanar geometry significantly improves our ability to filter the hologram in the spatial frequency domain $\left(f_{x}, f_{y}\right)$. The spatial frequency shift has both vertical and horizontal components, thereby moving the real and virtual images away from the $\mathrm{f}_{\mathrm{x}}=$ 0 and $\mathrm{f}_{\mathrm{y}}=0$ axes where the saturating autocorrelation content is located. Fig. 2(b) compares the Fourier spectra of simulated azimuth-only and azimuth-elevation holograms, illustrating the improved separation of the real image, the virtual image, and the autocorrelation terms in the spatial frequency domain for the latter. Lastly, the receiver boresight orientation was set at $\theta / 2$ to avoid standing wave reflections and to ensure that the receiver gain was approximately the same (within $1 \mathrm{~dB}$ ) for radiation incident from the object and reference beams. A standard $20 \mathrm{~dB}$ gain horn would have rejected these signals since they arrive more than 10 degrees from boresight.

\section{Hologram Simulations AND MEASUREMENTS}

The hologram acquisition and reconstruction processes for both samples were simulated to provide intuition and guide interpretation of the measurements. The simulation included all physical dimensions and refractive indices of the samples, the propagation of the object and reference beams, and the estimated image resolution of the holographic imager (which affects the space bandwidth product of the image).

The simulated image resolution was approximated with the Rayleigh criterion

$$
r=\frac{0.61 \lambda}{N A}
$$

where $\lambda$ is the wavelength, and $N A$ is the numerical aperture defined by image aperture and the sample-to-image plane distance $D$. For $D=163 \mathrm{~mm}$ and a $20 \times 20 \mathrm{~cm}$ image, $r=0.707 \mathrm{~mm}$ and $0.493 \mathrm{~mm}$ at $0.495 \mathrm{THz}$ and $0.710 \mathrm{THz}$, respectively. Accordingly, the imager's simulated pixel size was set to 0.707 and $0.493 \mathrm{~mm}$ for 0.495 and $0.710 \mathrm{THz}$, respectively.

\section{A. Sample A}

The sample A transfer function $t(x, y)$ may be formulated as

$$
t_{m}(x, y)=\exp \left(-\alpha_{m} \cdot L_{m}\right) \cdot \exp \left(i \cdot \frac{2 \pi}{\lambda} \cdot\left(n_{m}-1\right) L_{m}\right)
$$

or

$$
\begin{aligned}
& t_{m+s}(x, y) \\
& =\exp \left(-\frac{2}{3} \cdot \alpha_{m} \cdot L_{m}+\alpha_{s} \cdot L_{s}\right) \\
& \quad \cdot \exp \left(i \cdot \frac{2 \pi}{\lambda} \cdot\left(\frac{2}{3} \cdot\left(n_{m}-1\right) L_{m}+\left(n_{s}-1\right) L_{s}\right)\right)
\end{aligned}
$$



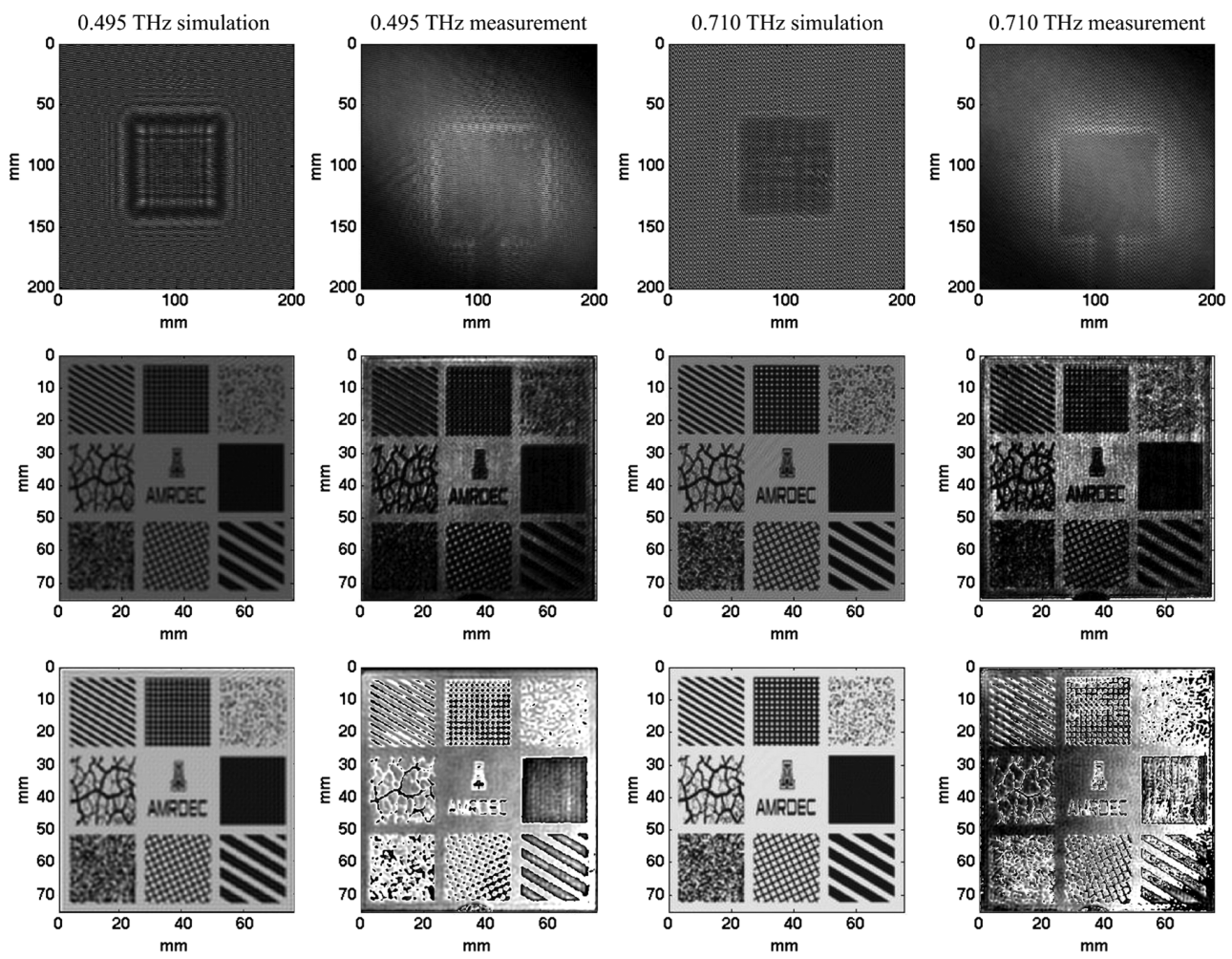

Fig. 3. Compilation of simulated and measured holograms, amplitude, and phase reconstructions (top to bottom rows) at $0.495 \mathrm{THz}$ (first two columns: simulated and measured) and $0.710 \mathrm{THz}$ (last two columns). In these grey scale amplitude (phase) images, black and white represent minimum $(-\pi)$ and maximum $(+\pi)$ transmission (phase), respectively.

where $L_{m}=3 \mathrm{~mm}$ and $L_{s}=1 \mathrm{~mm}$ are the thicknesses of the entire sample and embedded support material structures, respectively, and $\alpha$ and $\mathrm{n}$ are given in Table I. The choice of $t(x, y)$ depends on whether the z-dimension of $t(x, y)$ included support material $\left(t_{m+s}(x, y)\right)$ or was only made of model material $\left(t_{m}(x, y)\right)$. Sample A was simulated as a dielectric tranmissive and absorptive resolution chart. Reflection at each model/support material interface was omitted in this approximation for two reasons: the tiny difference in the real refractive indices of the model and support material $\left(10^{-2}\right.$, producing a void finesse $<10^{-4}$ ) and the strong absorption by the support material. Fig. 3 shows the simulated holograms with the amplitude and phase reconstructions.

As expected, the reconstructed images at $0.710 \mathrm{THz}$ are better resolved than those at $0.495 \mathrm{THz}$. A quantitative representation of the lateral image resolution is the modulation transfer function (MTF). The MTF of an optical system is analogous to a spatial frequency filter and was computed as the ratio of the spatial frequency content of the recorded image to that of a perfect imager. The spatial frequency map of a perfect imager is equivalent to the frequency content of the sample mask that was used to fabricate the sample. Matlab's two dimensional discrete Fourier transform algorithm $\mathrm{fft} 2(\mathrm{x}, \mathrm{y})$ was used to calculate all spatial frequency maps. Plots of the simulated and measured MTF of column 1, row 2 of the embedded structure are shown in Fig. 4. The MTFs for $0.495 \mathrm{THz}$ and $0.710 \mathrm{THz}$ are comparable from 0 to 0.7 cycles $/ \mathrm{mm}$, which marks the theoretical cutoff frequency for $0.495 \mathrm{THz}$ radiation. At that point the MTFs for $0.495 \mathrm{THz}$ drop below the MTF for $0.710 \mathrm{THz}$. The MTFs for $0.710 \mathrm{THz}$ decreases rapidly around its cutoff frequency of 1.0 cycles $/ \mathrm{mm}$.

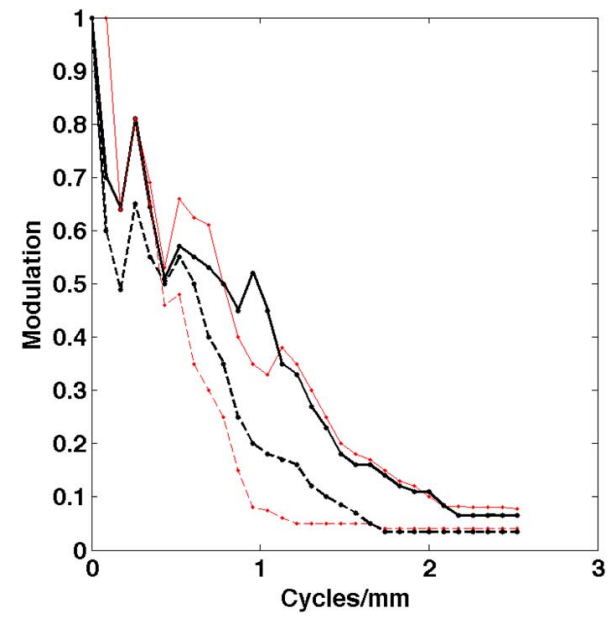

Fig. 4. Modulation transfer functions from the simulations (thin red) and measurements (thick black) of the first column, second row embedded structure of sample A at $0.495 \mathrm{THz}$, (dashed) and $0.710 \mathrm{THz}$ (solid).

The simulated and measured MTFs are in general agreement with each other.

\section{B. Sample B}

Sample B included air voids to maximize the index mismatch at the void interfaces, producing a void Finesse of 0.93 . The transmittance through sample B was simulated using the matrix theory of multilayer optics by sectioning the sample into four dielectric boundary matrices $\left(M_{1}, M_{3}, M_{5}, M_{7}\right)$ and three prop- 

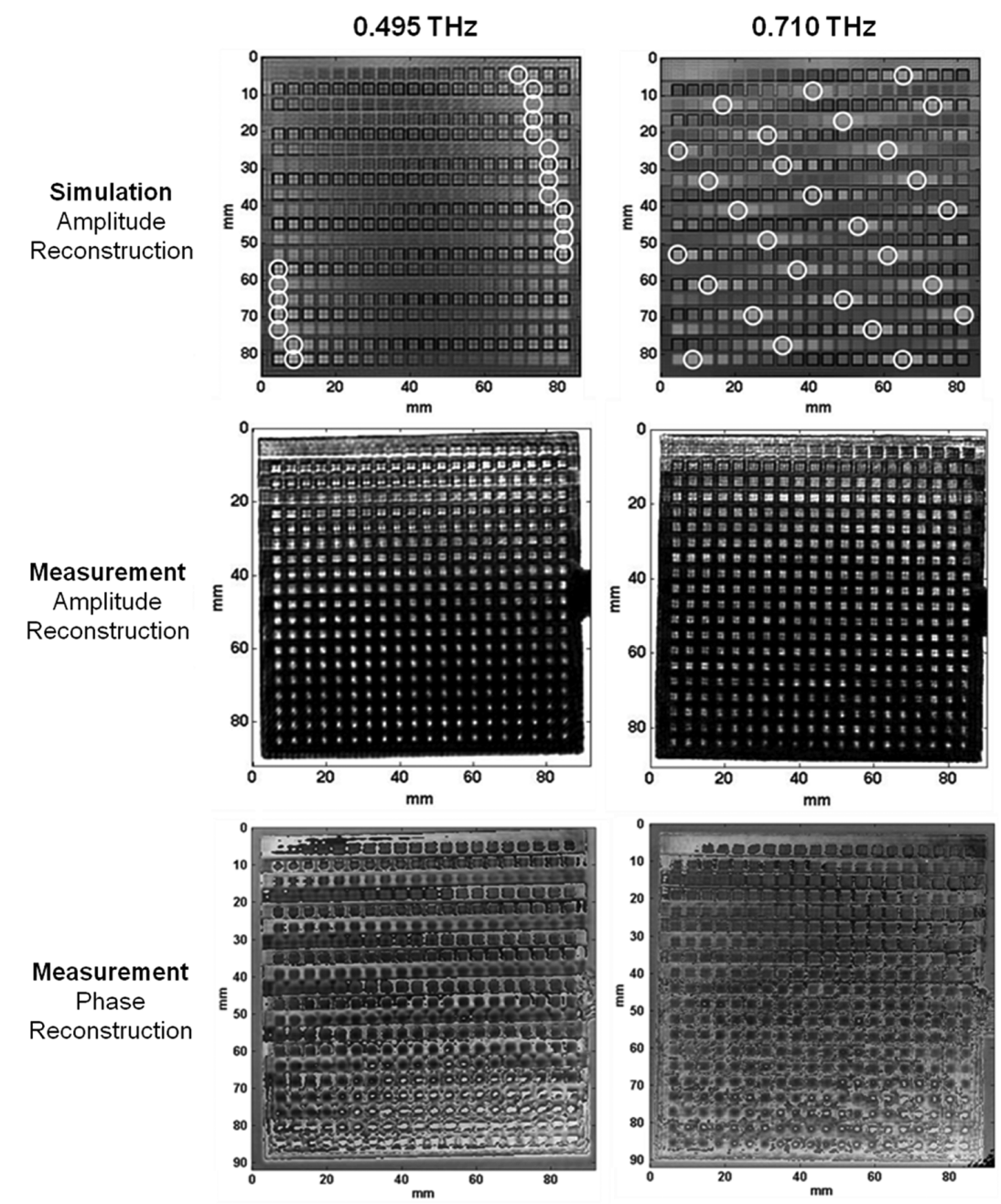

Fig. 5. Simulated (top) and measured amplitude transmittance patterns (middle), and measured phase transmittance pattern (bottom) at $0.495 \mathrm{THz}$ (left) and 0.710 $\mathrm{THz}$ (right) after hologram acquisition and reconstruction of sample B. In these grey scale amplitude (phase) images, black amd white represent minimum ( $-\pi$ ) and maximum $(+\pi)$ transmission (phase), respectively.

agation matrices $\left(M_{2}, M_{4}, M_{6}\right)[18]$. The boundary and propagation matrices have the general form

$$
\begin{aligned}
M_{\text {boundary }} & =\frac{1}{2 n_{2}}\left[\begin{array}{cc}
n_{2}+n_{1} & n_{2}-n_{1} \\
n_{2}-n_{1} & n_{2}+n_{1}
\end{array}\right] \\
M_{\text {propagation }} & =\left[\begin{array}{cc}
\exp (-i \delta) & 0 \\
0 & \exp (i \delta)
\end{array}\right]
\end{aligned}
$$

where $\delta=2 \pi \cdot n d / \lambda_{0}, n$ is the refractive index from Table $\mathrm{I}, \lambda_{0}$ is the free space wavelength, and $d$ is the propagation distance. The total wave transfer matrix $M_{\text {total }}$

$$
M_{\text {total }}=M_{7} M_{6} M_{5} M_{4} M_{3} M_{2} M_{1}=\left[\begin{array}{cc}
A & B \\
C & D
\end{array}\right]
$$

was converted to the scattering matrix

$$
S=\left[\begin{array}{ll}
t_{17} & r_{71} \\
r_{17} & t_{71}
\end{array}\right]=\frac{1}{D}\left[\begin{array}{cc}
A D-B C & B \\
-C & 1
\end{array}\right]
$$

where the recorded transmittance through the entire structure is equivalent to $\mathrm{t}_{17}$ or $(A D-B C) / D$. Hence analogous to (2) for sample $\mathrm{A}, t_{17}$ was used as the transfer function for sample B.
Fig. 5 shows the simulated transmission amplitude, the measured transmission amplitude, and the measured transmission phase at $0.495 \mathrm{THz}$ and $0.710 \mathrm{THz}$. In the simulated transmission amplitude, voids with Fabry-Pérot resonances producing transmission maxima for a given source frequency are marked with white circles. By contrast, the transmittance for some offresonant voids approaches that of the surrounding model material, making them almost indistinguishable from the host and therefore undetectable in an unknown object.

The accompanying reconstructed amplitude and phase images of sample B measured at 0.495 and $0.710 \mathrm{THz}$ are shown below the simulations in Fig. 5. Here, the amplitude values from void to void are related by the transmittance function modulated by the void resonances. These measured resonances are not as obvious as in the simulation, in part because the complex object and reference wavefields are not uniform and the samples may not have been perfectly perpendicular to the object beam. More importantly, the phase images at the bottom of Fig. 5 show that voids as thin as $45 \mu \mathrm{m}$ (third cell in first row), respectively $\lambda / 13$ and $\lambda / 9$ for 0.495 and $0.710 \mathrm{THz}$, can be resolved to within $\lambda / 40$ using this holographic technique [4]. This $\sim 10 \mu \mathrm{m}$ axial resolution—superior to what can be 
achieved with state-of-the-art time domain techniques-may ultimately be improved to the $\lambda / 100$ level typically observed of optical interferometry [16], [19]. The lateral resolution, which was sufficient to render easily recognizable images, was limited by the numerical aperture of the imager and was quantatively described by the MTF calculation for sample A.

In the measured amplitude images, the voids appear to become physically smaller towards the bottom of the sample where the deepest voids are located. This is due to the constraints imposed when amplitude images of very high dynamic range are plotted on a linear scale. Note that the phase images better capture the cavity dimensions because their constrained dynamic range repeats every $2 \pi$ radians. We hypothesize that the deeper voids act as lossy waveguides in which radiation near the sidewalls undergoes a combination of absorption, diffraction, and scattering, all of which decrease the transmittance. This effect is not visible in the simulation primarily because of two approximations. Firstly, the incident object beam was assumed to be planar traveling in the z-direction, but the sample was not aligned perfectly normal to the beam. Secondly, the diffraction pattern resulting from the interactions between the incident beam and object was assumed to originate from single beam-object interactions. Therefore secondary interactions, such as lateral cell wall reflections which significantly reduce the transmitted signal through the deep voids, are not included. The measurements show that only the fraction of radiation normally incident on the central area of each void propagates through the deeper voids.

Nevertheless, by plotting the transmission through the center of each of the 400 voids (Fig. 6), the expected spatial variation of these resonances emerges, especially when compared to simulated plots using $t_{17}$ from the matrix theory (3-5) approach. A Fourier analysis of these 400 voids shown in the insets of Fig. 6 reveals clearly that the cyclical, void-induced transmission variations are indeed present in the measurements. These Fabry-Pérot resonances occur with a periodicity that matches the predictions of the simulated transmittance plots: 3.1 cycles $/ \mathrm{mm}$ at $0.495 \mathrm{THz}$ and 4.3 cycles $/ \mathrm{mm}$ at $0.710 \mathrm{THz}$, where cycles $/ \mathrm{mm}$ represents the the number of resonances that occur in these increasingly thick voids per millimeter. The Fourier components from the simulated images are stronger than from the measured images because the simulation does not account for multi-path interference using a full three-dimensional diffraction model of the sample. The measured images are degraded by noise, alignment imperfections, and fabrication inaccuracies, but the general behavior is well reproduced. Additional low frequency content in the measured data was caused by alignment errors and residual amplitude variations that could not be removed through the flat-fielding process. This analysis of the transmissivity provides information on the relative depth variation between each void. Actual depths could also have been estimated if the thickness of the dielectric boundary had not been the same for each void.

In conclusion, we have demonstrated THzDH using an offaxis configuration to improve amplitude and phase resolution in order to locate and map voids in visually opaque dielectric media. Two 3D printed dielectric samples were investigated, one containing a variety of absorbant-filled void structures of slightly different dielectric constant, the other containing arrays
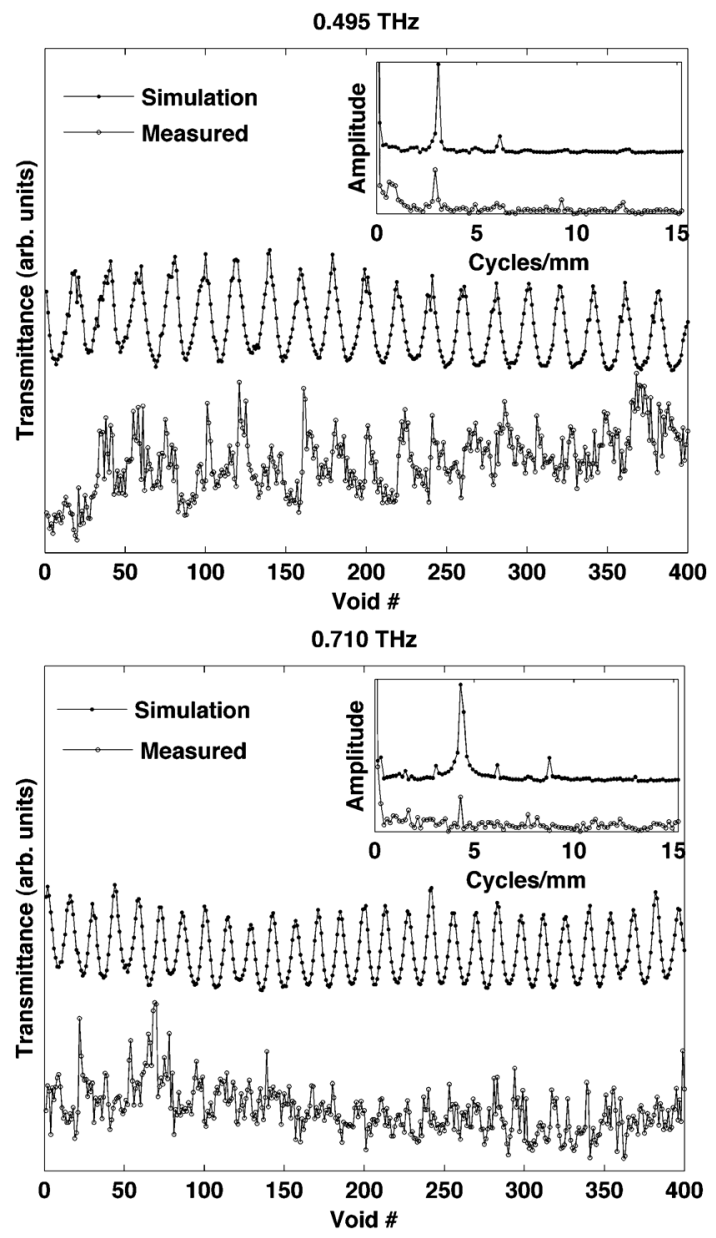

Fig. 6. Transmittance and spatial frequency plots (insets) of sample B's Fabry-Pérot resonance pattern simulation and measurements at 0.495 and $0.710 \mathrm{THz}$.

of empty square voids of increasing depth. We find that a simple transfer matrix model intended to guide the interpretation of the measurements captures the dominant scattering behaviors except for the deepest voids. The measured wavelength-dependent lateral resolution was consistent with the simulated MTF for the ideal target, and the $\sim \lambda / 40$ axial resolution is consistent with traditional optical holographic imaging. The methodology presented here may be employed for rapid, high resolution holographic imaging when arrays of sensitive $\mathrm{THz}$ detectors become available.

\section{REFERENCES}

[1] D. Parshall, "Digital holographic microscopy with dual-wavelength phase unwrapping," Appl. Opt., vol. 45, no. 3, pp. 451-459, Jan. 2006.

[2] U. Schnars and W. Jueptner, Digital Holography: Digital Hologram Recording, Numerical Reconstruction, and Related Techniques, 1st ed. Berlin, Germany: Springer, 2010.

[3] M. K. Kim, L. Yu, and C. J. Mann, "Interference techniques in digital holography," J. Opt. A: Pure Appl.Opt., vol. 8, pp. S518-S523, Jun. 2006.

[4] M. S. Heimbeck, M. K. Kim, D. A. Gregory, and H. O. Everitt, "Terahertz digital holography using angular spectrum and dual wavelength reconstruction methods," Opt. Express, vol. 19, no. 10, pp. 9192-9200, May 2011.

[5] S.-H. Ding, Q. Li, Y.-D. Li, and Q. Wang, "Continuous-wave terahertz digital holography by use of a pyroelectric array camera," Opt. Lett., vol. 36, no. 11, pp. 1993-1995, May 2011. 
[6] X. Wang, W. Xiong, W. Sun, and Y. Zhang, "Coaxial waveguide mode reconstruction and analysis with THz digital holography," Opt. Express, vol. 20, pp. 7706-7715, 2012.

[7] J. Gass and A. Dakoff, "Phase imaging without $2 \pi$ ambiguity by multiwavelength digital holography," Opt. Lett., vol. 28, no. 13, July 2003.

[8] C. F. Cull, D. A. Wikner, J. N. Mait, M. Mattheiss, and David J. Brady, "Millimeter-wave compressive holography," Apl. Opt., vol. 49, no. 19, pp. E67-E82, July 2010.

[9] B. Scherger, C. Joerdens, and M. Koch, "Liquid-filled variable-focus terahertz lens," presented at the Optical Terahertz Science and Technology Conf., Santa Barbara, CA, USA, 2011.

[10] M. S. Heimbeck, P. Reardon, J. Callahan, and H. O. Everitt, "Transmissive quasi-optical Ronchi phase grating for terahertz frequencies," Opt. Lett., vol. 35, no. 21, pp. 3658-3660, Nov. 2010.

[11] K. B. Cooper et al., "Penetrating 3-D imaging at 4- and 25-m range using a submillimeter-wave radar," IEEE Trans. Microw. Theory Techn., vol. 56, no. 12, pt. 1, pp. 2771-2778, Dec. 2008.

[12] M. S. Heimbeck, D. L. Marks, D. Brady, and H. O. Everitt, "Terahertz interferometric synthetic aperture tomography for confocal imaging systems," Opt. Lett., vol. 37, pp. 1316-1318, 2012.

[13] "3D-printing materials specifications," in SIGGRAPH 2013 [Online]. Available: http://www.karlddwillis.com/wp/wp-content/ uploads/2011/03/SIGGRAPH2013InfraStructs.pdf

[14] Z. Wu, W.-R. Ng, M. E. Gehm, and H. Xin, "Terahertz electromagnetic crystal waveguide fabricated by polymer jetting rapid prototyping," Opt. Express, vol. 19, pp. 3962-3972, 2011.

[15] M. E. Gehm, Z. Wu, and H. Xin, "Rapid prototyping for fabrication of GHz-THz bandgap structures," THz Physs., vol. 7311, p. 10, 2009.

[16] K. D. D. Willis and A. D. Wilson, "Infrastructs: Fabricating information inside physical objects for imaging in the terahertz region," $A C M$ Trans. Graphics, vol. 32, no. 4, Jul. 2013, article 138.

[17] J. W. Goodman, Introduction to Fourier Optics, 3rd ed. Englewood, CO, USA: Roberts \& Co., 2005.

[18] B. E. A. Saleh and M. C. Teich, Fundamentals of Photonics, 2nd ed. Hobokem, NJ, USA: Wiley, 2007, Wiley Series in Pure and Applied Optics.

[19] E. Vikhagen, "TV holography: Spatial resolution and signal resolution in deformation analysis," Apl. Opt., vol. 10, no. 4, pp. 420-425, Feb. 1991.

Martin S. Heimbeck received the B.S. degree in physics from the University of North Alabama, Florence, AL, USA, in 2006, and the M.S. degrees in physics from The University of Alabama in Huntsville, Huntsville, AL, USA, in 2008.

$\mathrm{He}$ is currently working toward the Ph.D. degree in optical science and engineering from The University of Alabama in Huntsville, Huntsville, AL, USA, as an employee of the Charles M. Bowden Research Center in the Army's Aviation \& Missile RD\&E Center located at Redstone Arsenal, AL, USA. His research interests include coherent MMW/THz imaging and MMW radar applications.

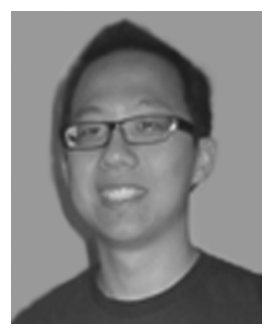

Wei-Ren Ng was born in Kuala Lumpur, Malaysia, in 1985. He received the B.S. and M.S. degrees in electrical engineering from the University of Arizona, Tucson, AZ, USA, in 2008 and 2012, respectively, and is currently working toward the Ph.D. degree (under Dr. Michael Gehm) from the same university.

Since 2008, he has served as a graduate research assistant in Dr. Michael Gehm's Non-traditional Sensors Laboratory. His research interests include 3D printed $\mathrm{MMW} / \mathrm{THz}$ components, $\mathrm{MMW} / \mathrm{THz}$ imaging, digital signal processing, image processing, holography and diffractive optics, spectroscopy, and biomedical engineering.

$\mathrm{Mr} . \mathrm{Ng}$ is a member of HKN and TBP.

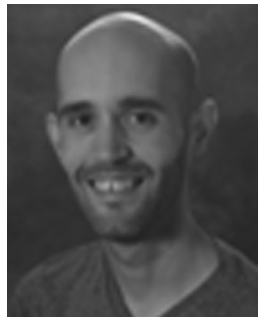

Dathon R. Golish was born in Mesa, AZ, USA, in 1979. He received B.S. degrees in astronomy and physics in 2002, and both the M.S. and Ph.D. degrees in optical sciences in 2008, all from the University of Arizona (UA), Tucson, AZ, USA.

From 2008 to 2010, he worked as a senior optical engineer at TeraVision Inc., a small UA-spinoff company. In 2010, he was a postdoctoral researcher in the Electrical and Computer Engineering department at UA. He became an assistant research professor in 2011 and continued to conduct research in the ECE department until 2013. Since 2013, he is a Calibration and Test Engineer for the OSIRIS-REx project under the Lunar and Planetary Laboratory, University of Arizona (UA), Tucson, AZ, USA

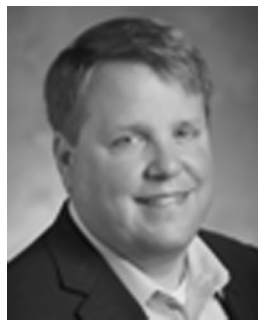

Michael E. Gehm was born in Würzburg Germany, in 1969. He received the B.S. degree in mechanical engineering from Washington University, St. Louis, MO, USA, in 1992, and the M.S. and Ph.D. degrees in physics from Duke University, Durham, NC, USA, in 1998 and 2003, respectively.

From 2003 to 2006 , he was a postdoctoral researcher in the Electrical and Computer Engineering Department at Duke University. From 2007 to 2013, he was an Assistant Professor in the Department of Electrical and Computer Engineering and the College of Optical Sciences at the University of Arizona, Tucson, AZ, USA In 2013, he joined the faculty of Duke University, Durham, NC, USA, as an Associate Professor of Electrical and Computer Engineering. His research interests include computational and compressive sensing, the rapid prototyping of functional electromagnetic components, and optical physics.

Henry O. Everitt was born in Huntsville, AL, USA, in 1963. He received the B.S., M.S., and Ph.D. degrees in physics from Duke University, Durham, NC, USA, in 1985, 1987, and 1990, respectively.

From 1991 to 2005 , he was a program manager and eventually chief scientist of the Army Research Office where he managed programs in condensed matter physics, nanotechnology, and quantum information science. He joined the Army's Aviation \& Missile Research, Development, and Engineering Center in 2005 as a senior research scientist. Since 1994, he has served as an adjunct professor of physics at Duke University. He is the author of four book chapters and more than 100 articles. His research interests include ultrafast spectroscopic analysis of wide bandgap semiconductors and nanostructures, nanoplasmonics, inorganic phosphors, molecular spectroscopy, remote sensing, optically pumped $\mathrm{THz}$ lasers, and coherent MMW/THz imaging.

Dr. Everitt is a fellow of the American Physical Society and the Optical Society of America, and is an emeritus fellow of the Army Research Laboratory. 